\title{
A cluster randomized trial of delivery of intermittent preventive treatment of malaria in pregnancy at the community level in Burkina Faso
}

Julie R. Gutman ${ }^{1 *} \mathbb{D}$, Daniel K. Stephens², Justin Tiendrebeogo ${ }^{3}$, Ousmane Badolo³, Mathurin Dodo33, Danielle Burke ${ }^{4}$, John Williamson ${ }^{1}$, Kristen Vibbert ${ }^{4}$, Susan J. Youll ${ }^{5}$, Yacouba Savadogo ${ }^{6}$ and William R. Brieger ${ }^{1}$

\begin{abstract}
Background: Malaria in pregnancy is responsible for $8-14 \%$ of low birth weight and $20 \%$ of stillbirths in sub-Saharan Africa. To prevent these adverse consequences, the World Health Organization recommends intermittent preventive treatment of pregnant women (IPTp) with sulfadoxine-pyrimethamine be administered at each ANC visit starting as early as possible in the second trimester. Global IPTp coverage in targeted countries remains unacceptably low. Community delivery of IPTp was explored as a means to improve coverage.
\end{abstract}

Methods: A cluster randomized, controlled trial was conducted in 12 health facilities in a 1:1 ratio to either an intervention group (IPTp delivered by CHWs) or a control group (standard practice, with IPTp delivered at HFs) in three districts of Burkina Faso to assess the effect of IPTp administration by community health workers (CHWs) on the coverage of IPTp and antenatal care (ANC). The districts and facilities were purposively selected taking into account malaria epidemiology, IPTp coverage, and the presence of active CHWs. Pre- and post-intervention surveys were carried out in March 2017 and July-August 2018, respectively. A difference in differences (DiD) analysis was conducted to assess the change in coverage of IPTp and ANC over time, accounting for clustering at the health facility level.

Results: Altogether 374 and 360 women were included in the baseline and endline surveys, respectively. At baseline, women received a median of 2.1 doses; by endline, women received a median of 1.8 doses in the control group and 2.8 doses in the intervention group ( $p$-value $<0.0001$ ). There was a non-statistically significant increase in the proportion of women attending four ANC visits in the intervention compared to control group ( $\mathrm{DiD}=12.6 \%, \mathrm{p}$-value $=0.16$ ). By the endline, administration of IPTp was higher in the intervention than control, with a DiD of 17.6\% for IPTp3 (95\% confidence interval (Cl) - 16.3, 51.5; p-value 0.31) and 20.0\% for IPTp4 $(95 \% \mathrm{Cl}-7.2,47.3$; p-value $=0.15)$.

Conclusions: Community delivery of IPTp could potentially lead to a greater number of IPTp doses delivered, with no apparent decrease in ANC coverage.

Keywords: Malaria, Pregnancy, Intermittent preventive treatment, Sulfadoxine-pyrimethamine

\footnotetext{
*Correspondence: fff2@cdc.gov

${ }^{1}$ Malaria Branch, Division of Parasitic Diseases and Malaria, Center for Global Health, US Centers for Disease Control and Prevention, 1600 Clifton Road, Mailstop H24-3, Atlanta, GA 30329, USA

Full list of author information is available at the end of the article
}

\section{Background}

Despite the considerable decline in malaria since 2000, malaria in pregnancy (MIP) remains a major public health problem. MIP is associated with maternal anaemia, preterm deliveries, low birth weight, and an increased risk of neonatal death [1]. Reference before punctuation, please

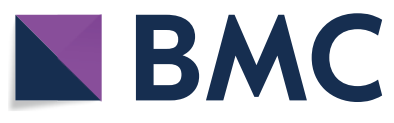

(c) The Author(s) 2020. This article is licensed under a Creative Commons Attribution 4.0 International License, which permits use, sharing, adaptation, distribution and reproduction in any medium or format, as long as you give appropriate credit to the original author(s) and the source, provide a link to the Creative Commons licence, and indicate if changes were made. The images or other third party material in this article are included in the article's Creative Commons licence, unless indicated otherwise in a credit line to the material. If material is not included in the article's Creative Commons licence and your intended use is not permitted by statutory regulation or exceeds the permitted use, you will need to obtain permission directly from the copyright holder. To view a copy of this licence, visit http://creativeco mmons.org/licenses/by/4.0/. The Creative Commons Public Domain Dedication waiver (http://creativecommons.org/publicdomain/ zero/1.0/) applies to the data made available in this article, unless otherwise stated in a credit line to the data. 
change throughout to prevent the adverse effects of MIP, in areas of moderate to high malaria transmission, the World Health Organization (WHO) recommends the use of long-lasting insecticide-treated nets (LLINs), intermittent preventive treatment of malaria in pregnancy (IPTp) with sulfadoxine pyrimethamine (SP), and prompt and effective treatment of pregnant women, targeting $100 \%$ coverage among at risk populations [2-4].

Since 2012, the WHO has recommended that pregnant women receive IPTp-SP as early as possible starting at the beginning of the second trimester (13th week of pregnancy), at every antenatal care (ANC) contact (which typically occurs in health facilities) up until delivery, with doses spaced at least 1 month apart, ideally administering a minimum of three doses during pregnancy (IPTp3) [5]. Despite this recommendation, which was meant to increase IPTp uptake to 3 or more, coverage remains low. In 2017, among 33 African countries, an estimated 22\% of pregnant women received the recommended three doses, and only $42 \%$ received two doses [6]. Novel strategies are needed to improve uptake.

Several studies have suggested that community health workers (CHWs) might be able to effectively deliver IPTp while at the same time promoting ANC attendance at health facilities [7-11]. Two studies in Nigeria and Uganda utilized CHWs who were integrated with local health services and trained to deliver IPTp and refer women to ANC. These studies improved uptake of at least two doses of IPTp (by 35.3 percentage-points in Nigeria and 37.3 percentage-points in Uganda); in Nigeria there was no effect on ANC attendance, while in Uganda, mean ANC attendance was 3.3 visits in the intervention arm and only 2.6 in the control arm $(\mathrm{p}<0.001)$ [ 8]. Two studies in Uganda and Malawi trained a variety of community-based agents to deliver IPTp, without explicitly seeking to increase ANC attendance, and while IPTp uptake improved (27.6 and 29.3 percentage point difference in Uganda and Malawi, respectively), ANC attendance did not (19.3 and 17.9 percentage point lower ANC attendance in the community delivery arm in Uganda and Malawi, respectively) $[9,10]$. A prior study in Burkina Faso used female community volunteers to encourage both ANC attendance and IPTp uptake through ANC, resulting in increased ANC attendance (19.5 percentage points higher in the community promotion arm) and IPTp uptake (22.7 percentage points higher in the community promotion arm) [11].

In its 2016-2020 National Malaria Strategic Plan, Burkina Faso set a goal of $100 \%$ of pregnant women receiving three doses of IPTp [4]. However, coverage of IPTp3 remains below national and international targets at $57.7 \%$ [12], despite high rates of ANC attendance (95\% of women attend at least once and $33.7 \%$ attend four times)
[13]. Thus, the Ministry of Health (MOH) is exploring alternative approaches to providing IPTp, including community level distribution of IPTp (cIPTp).

\section{Methods}

A cluster randomized, controlled trial assessing the effect of IPTp administration by CHWs (compared to the routine practice of delivering IPTp at health facilities) on the coverage of IPTp and ANC was conducted in three districts of Burkina Faso over 15 months, from May 2017 to August 2018, with baseline and post-intervention surveys carried out in March 2017 and July-August 2018, respectively.

\section{Study area}

With an estimated population of 20 million, Burkina Faso is a high malaria endemic Sahelian country in the heart of West Africa accounting for $4 \%$ of the world's annual malaria cases [14]. Women comprise $51.8 \%$ of the overall population, and it is expected that $5.5 \%$ of women will be pregnant at any given time [15]. The study was implemented in three of the 13 regions (Sud-Ouest [SouthWest], Centre-Sud [Central-South], and Centre-Est [Central-East]) with highest malaria transmission. One district was purposively selected from each of the three regions, taking into account malaria epidemiology, IPTp coverage, and the presence of active CHWs (Fig. 1). Routine HMIS data indicate that before the trial, IPTp3 coverage in the selected districts, Batié, Pô, and Ouargaye, was $34.6 \%, 52.8 \%$, and $47.2 \%$, respectively, for an overall average of $44.9 \%$.

Non-contiguous health facilities (HF) in each district were matched by estimated general population and estimated number of pregnant women and IPTp2 coverage (based on routine health facility data). In each district, two pairs of HFs were purposively selected, and one HF in each pair was randomly assigned to the intervention, for a total of $12 \mathrm{HFs}$ (six intervention, six control) (Table 1).

The study intervention included all pregnant women living in the targeted areas, while the baseline and endline surveys included women aged 18-49 years in selected households who had given birth in the 9 months prior to each survey. The catchment area of these HFs included 113 villages, all of which were included in the cross-sectional survey, with sample size per village selected proportional to village size. At the village level, the Expanded Programme on Immunization (EPI) sampling method was used to select households where women would be interviewed [16]. At the center of the village, a direction was chosen by spinning a bottle on the ground. Households along the indicated path were visited and the 


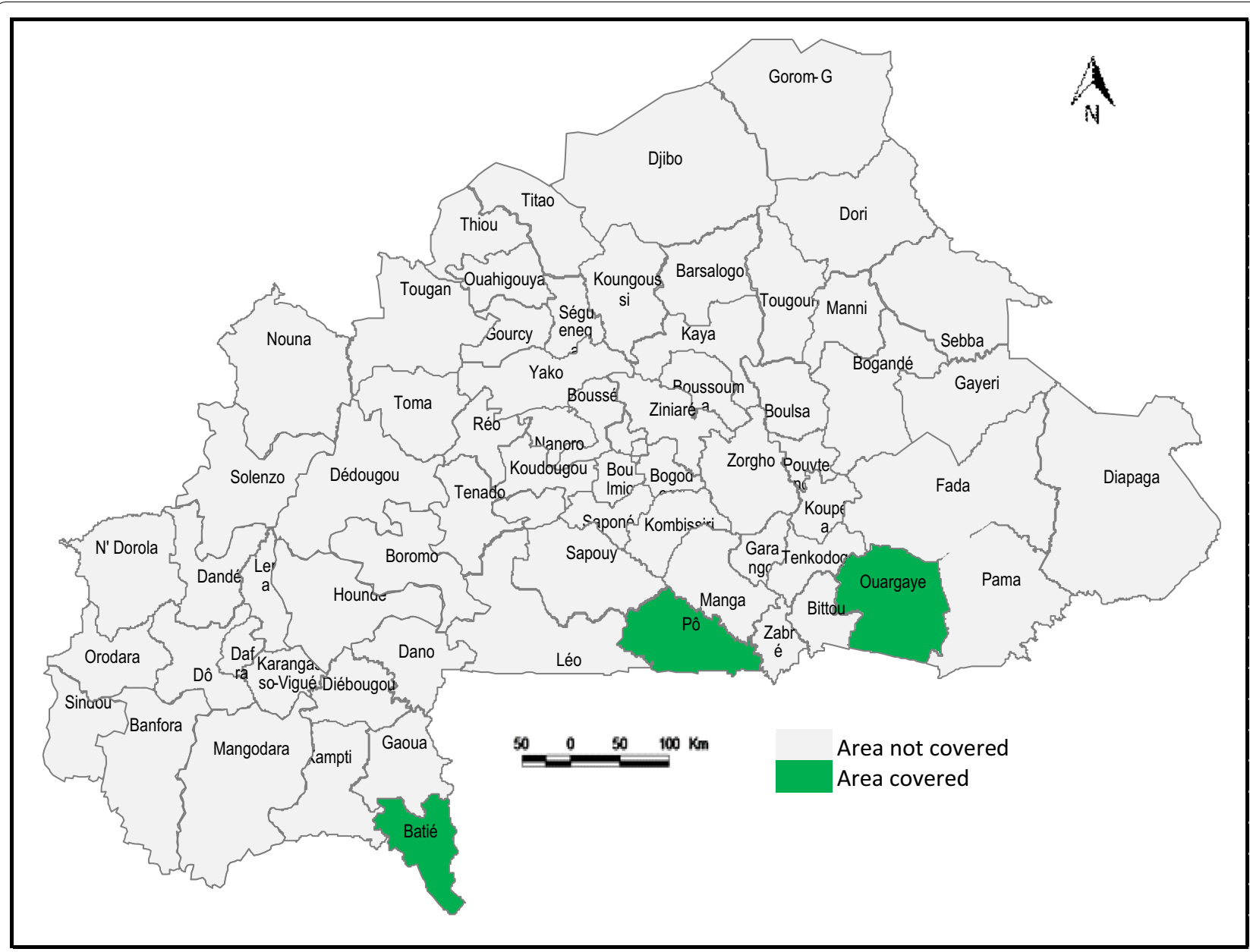

Fig. 1 Map of Burkina Faso showing the three districts involved in the study

Table 1 Characteristics of included facilities

\begin{tabular}{|c|c|c|c|c|c|c|}
\hline District & Study arm & Health facility & Total population & $\begin{array}{l}\text { Number } \\
\text { of villages }\end{array}$ & $\begin{array}{l}\text { Number of community } \\
\text { health workers }\end{array}$ & $\begin{array}{l}\text { Number } \\
\text { of expected births } \\
\text { annually }\end{array}$ \\
\hline \multirow[t]{4}{*}{ Batié } & Intervention & Dankana & 6991 & 21 & 42 & 379 \\
\hline & Control & Midebdo & 7648 & 30 & 60 & 417 \\
\hline & Intervention & Boussoukoula & 5484 & 16 & 32 & 297 \\
\hline & Control & Tamipar & 6078 & 16 & 32 & 332 \\
\hline \multirow[t]{4}{*}{ Pô } & Intervention & Guiaro & 9001 & 8 & 8 & 490 \\
\hline & Control & Kaya & 8408 & 6 & 6 & 458 \\
\hline & Intervention & Kampala & 7760 & 8 & 8 & 423 \\
\hline & Control & Guelwongo & 8757 & 3 & 3 & 477 \\
\hline \multirow[t]{4}{*}{ Ouargaye } & Intervention & Nabangou & 5770 & 4 & 12 & 334 \\
\hline & Control & Salembore & 6467 & 2 & 12 & 374 \\
\hline & Intervention & Konglore & 3490 & 2 & 6 & 202 \\
\hline & Control & Tensobtenga & 4012 & 1 & 8 & 232 \\
\hline
\end{tabular}


questionnaire was administered to consenting, eligible women until the desired sample size was achieved.

\section{Sample size}

A sample size of 360 women (30 women per health facility catchment area) who had delivered in the last 9 months was required for each survey to achieve $80 \%$ power to detect a difference between the group proportions of approximately $19.6 \%$, from a baseline proportion of $44.7 \%$ (average proportion in the three districts) to an endline proportion of $64.3 \%$ in the intervention arm, as estimated using PASS V14 (NCSS, LLC, Kaysville, UT) to assess sample size for cluster randomized trial, at a significance level of 0.05 and assuming an intra-cluster correlation (ICC) of 0.03 .

\section{Implementation process}

The CHW registers were designed to provide a reminder that IPTp should be given monthly, and not more frequently, and to help the CHW reconcile who needed follow-up. Existing male CHWs were paired with female volunteers ("animatrices"), to ensure that home visits were only conducted by women, as there was concern that it would not be culturally acceptable for male CHWs to enter the homes and speak to pregnant women. If the female volunteer was not literate, the male $\mathrm{CHW}$ in that village assisted her in completing the forms, and at times accompanied her on the home visit. Formal CHWs received approximately \$35USD monthly from the government as compensation for their duties; female volunteers recruited by the study received the same amount in monthly compensation from the study.

The MOH ensured the availability of SP tablets/treatment for IPTp in both control and intervention HFs throughout the study, including for distribution by trained CHWs. Each pregnant woman seen at ANC in the intervention facility received information on the community IPTp study and had the choice to participate or not. Pregnant women who wanted to receive SP had their identities recorded on a summary sheet and in CHW registers to facilitate their follow-up for the intervention. Women were asked to follow-up with the CHW monthly; those who did not present for the scheduled visit with the $\mathrm{CHW}$ were followed at home. The first dose of IPTp-SP was always given as directly observed therapy (DOT) at the HF to ensure that a health provider confirmed the woman's gestational age and initiated IPTp after the end of the first trimester. CHWs also provided IPTp-SP as directly observed therapy, as part of their monthly household visits starting with the second dose, provided that SP had not been administered in the preceding 4 weeks. This was then recorded on the woman's ANC card, which had space for recording up to five doses. CHWs also encouraged women to attend ANC, collected data on any adverse events, and referred women to the facility in the case of adverse events. CHW data were collected monthly. CHWs were supervised monthly by HF workers; monthly meetings were facilitated by the study.

\section{Training}

Activities were implemented using the cascade training approach. The first training session included centrallevel stakeholders (National Malaria Control Programme, Directorate of Family Health, Directorate for Health Promotion and Education, Directorate of Sectorial Statistics) and the field coordinator from each district. The main objective of this training was to orient stakeholders on the organization and use of the various intervention tools. The stakeholder then transferred the information to the HF health workers, who in turn trained the CHWs (33 existing male and 25 existing female CHWs plus an additional 33 female volunteers).

For each survey, ten people were trained over 2 days in each district, and eight were chosen as field data collectors. The trainings focused on mastery of data collection tools, ethical rules, and ensuring data quality. CHWs and HF workers were trained on potential adverse events related to SP administration and instructed to report any adverse events to the study coordinator.

\section{Data analysis}

Routine service data (ANC visits, IPTp doses delivered by CHWs and at the HF) were summarized monthly to monitor the intervention. The primary outcome was the change in IPTp3 coverage over time assessed using a difference in difference analysis comparing the baseline and endline cross sectional survey data. In addition, a descriptive analysis of the socio-demographic characteristics of women included in the baseline and endline surveys, stratified by intervention and control areas, was performed. The REG procedure in SAS was used to assess for differences in medians. Difference in differences analyses were conducted on the survey data to assess the change over time in additional secondary outcomes, including any ANC visits (ANC1), four or more ANC visits (ANC4), IPTp1, 2, 4, and 5 coverage. Both primary and secondary outcomes were based on what was recorded on the ANC card, up to a maximum of five IPTp doses. The analyses used logistic regression with generalized estimating equations with the GENMOD procedure in SAS Version 9.4 (SAS Institute Inc., Cary, NC) for binary outcomes (ANC1, ANC4, IPTp3, IPTp4) with difference in differences calculated using a binary model with an identity link function, and Poisson regression for continuous outcomes (number of IPTp doses, number of ANC visits, timing of initiation of ANC). This 
was done by incorporating an intervention group variable, a variable indicating pre- or post-intervention, and an intervention-time interaction term. The ICC was calculated using the same model with the "corrw" option in the repeated statement. In order to obtain $95 \%$ confidence intervals $(95 \% \mathrm{CI})$ for the difference in differences, a linear model was run using the GENMOD procedure, including all the same terms as in the logistic model. All analyses accounted for clustering at the HF level. Maternal age and gravidity were included a priori in the model; district and maternal education were explored as covariates but dropped due to lack of significance. A p-value of less than 0.05 was considered statistically significant.

\section{Ethics}

The protocol was reviewed and approved by the John's Hopkins School of Public Health (JHSPH) and Burkina Faso Institutional Review Boards (IRB); the Centers for Disease Control and Prevention Human Subjects Office determined that CDC staff were not engaged in human subjects research. Verbal informed consent was obtained from each woman in the local language prior to data collection.

\section{Results}

\section{Routine data}

Overall, 9834 doses were delivered to 2266 women living in the intervention HF catchment areas, with 50\% of these given by CHWs, while 6683 doses were delivered to 2030 women living in the control HF catchment areas. There was improvement in the number of doses of IPTp delivered (Fig. 2), as well as in retention in care, measured by the number of women who completed four or more ANC visits out of the number of women who attended the first ANC visit (Fig. 3). During the period of the study, no adverse events related to SP administration were reported.

\section{Cross sectional survey results}

A total of 374 women were interviewed in the baseline survey and 360 in the endline survey. Socio-demographic characteristics of women in the control and intervention groups were similar both within and between surveys (Table 2). On average, a quarter of surveyed women had had one pregnancy, one-third had two to three pregnancies, and the rest had four or more.

At baseline, women received a median of 2.1 doses (range 0-5 doses) across both arms according to documentation on the ANC card, and a median of 2.5 doses (range 0-6) according to self-report, with no significant differences across arms. At endline, control group women received a median of 1.8 doses (range $0-5$ ) according to the card and 2.8 doses (range $0-7$ ) per self-report and women in the intervention group received 2.8 doses (range $0-5$ ) per ANC card and 3.4 (range $0-7$ ) per self-report (p-value $<0.0001$ for difference in arms by both card and self-report).

ANC4, IPTp3, and IPTp 4 coverage were similar at baseline, with ANC4 coverage of $61.8 \%$ in the intervention and $62.2 \%$ in the control ( $\mathrm{p}$-value $=0.96$ ), IPTp3 coverage of $50.5 \%$ in the intervention and $54.3 \%$ in the control ( $\mathrm{p}$-value $=0.74$ ), and IPTp4 coverage of $21.5 \%$ in the intervention and $16.0 \%$ in the control $(\mathrm{p}$-value $=0.59)($ Table 3$)$.

There was a 15.4 percentage point increase in the proportion of pregnant women who had attended at least four ANC visits in the intervention area, and a 2.8 percentage point increase in ANC4 attendance among women in the control group, for a $\mathrm{DiD}$ of 12.6 percentage points $(95 \% \mathrm{CI}-7.0,3.2)$. By the endline, administration of IPTp was universally higher in the intervention than control. There was an overall 17.6 percentage point increase in IPTp3 at endline in the intervention group, after accounting for baseline coverage $(\mathrm{DiD} 95 \% \mathrm{CI}-16.3,51.5$; $\mathrm{p}$-value $=0.31)$. There was a 25.2 percentage-point increase in IPTp4 in the intervention area (p-value 0.02 for comparison from baseline to endline), and only a 5.1 percentage-point increase in the control ( $\mathrm{p}$-value 0.60 for comparison from baseline to endline), with a difference in differences of $20.0 \%$ (95\% CI $-7.2,47.3$; p-value $=0.15)$. The greatest improvement was seen in IPTp5, which increased from $4.3 \%$ at baseline to $27.8 \%$ at endline in the intervention arm $(\mathrm{DiD}=23.9$ percentage points, 95\% CI 9.4, 38.4; $\mathrm{p}=0.001$ ) (Table 3). Results did not change substantially when adjusted for maternal age and gravidity (Table 4). The calculated ICC was 0.095 for IPTp3 and 0.089 for IPTp4. Gravidity was associated with receipt of IPTp3, but not IPTp4 nor ANC attendance, while age greater than 20 was associated with ANC attendance (both ANC1 and ANC4), but not receipt of IPTp (Table 5).

At baseline, women reported first presenting to ANC at a mean of 3.1 and 2.8 months gestational age, in control and intervention arms, respectively ( $\mathrm{p}$-value $=0.07$ ); while at endline, mean gestational age at first presentation to ANC was reported to be 3.0 and 2.6 months (p-value 0.03 ) in control and intervention arms, controlling for age and gravidity. Among the $90.1 \%$ of women who stated that they told someone about their pregnancy, the vast majority (91.7\%) disclosed that they were pregnant prior to feeling the baby moving/showing, with no significant differences between baseline/endline or control and intervention groups. Two-thirds (65.9\%) reported having first told their husbands/the father of the baby, $12.1 \%$ first told their mother in law, $8.2 \%$ first told a 


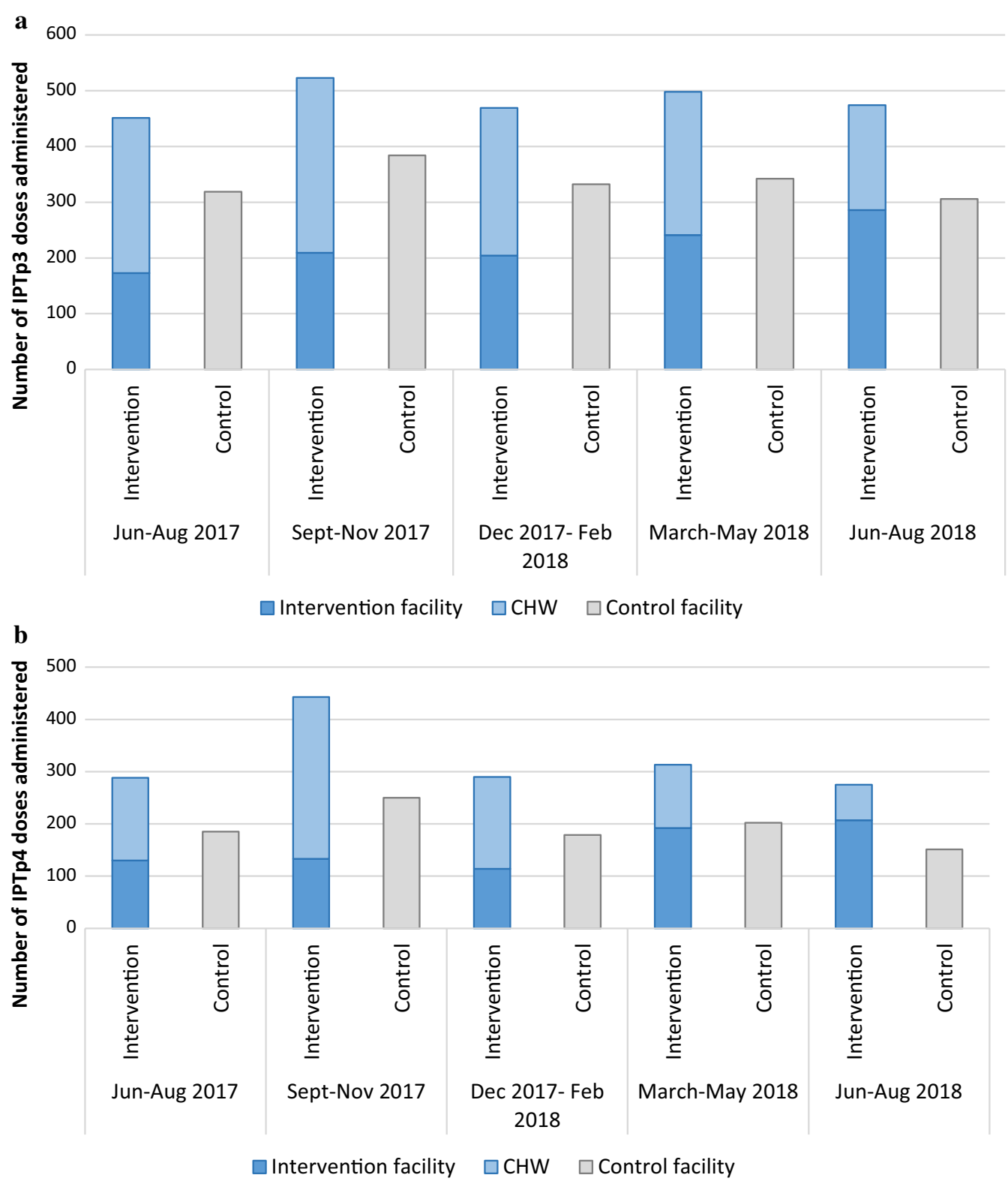

Fig. 2 Number of a IPTp3 and b IPTp4 doses administered by district and quarter from June 2017 to August 2018Coverage rates for IPTp3 (a) and IPTp4 (b) based on routine health facility and CHW data

HF health worker, and $4.9 \%$ first told their mother; only $1.8 \%$ first reported their pregnancy to a CHW.

Adjusting for age and gravidity, at baseline $34.4 \%$ and $56.7 \%$ pregnant women in the control and intervention arms, respectively, reported having spoken to a $\mathrm{CHW}$ about their pregnancy; this increased to $54.0 \%$ and $77.6 \%$ in the control and intervention arms, respectively, at endline. The increase was statistically significant in both arms ( $p$-value $<0.0001$ in control and p-value 0.0028 in the intervention arm; $\mathrm{p}$-value 0.0002 for the difference between arms at endline). Similar proportions reported discussing prevention of MIP with a CHW $(26.7 \%$ and $57.9 \%$ in the control and intervention arms, respectively, at baseline, and $51.3 \%$ and $74.3 \%$ in the control and intervention arms, respectively, at endline). The increase was statistically significantly different in the control ( $\mathrm{p}$-value 0.007 ) but not the intervention arm (p-value 0.13), however, given the difference at baseline there was still a statistically significant difference between arms at endline (p-value 0.01). 


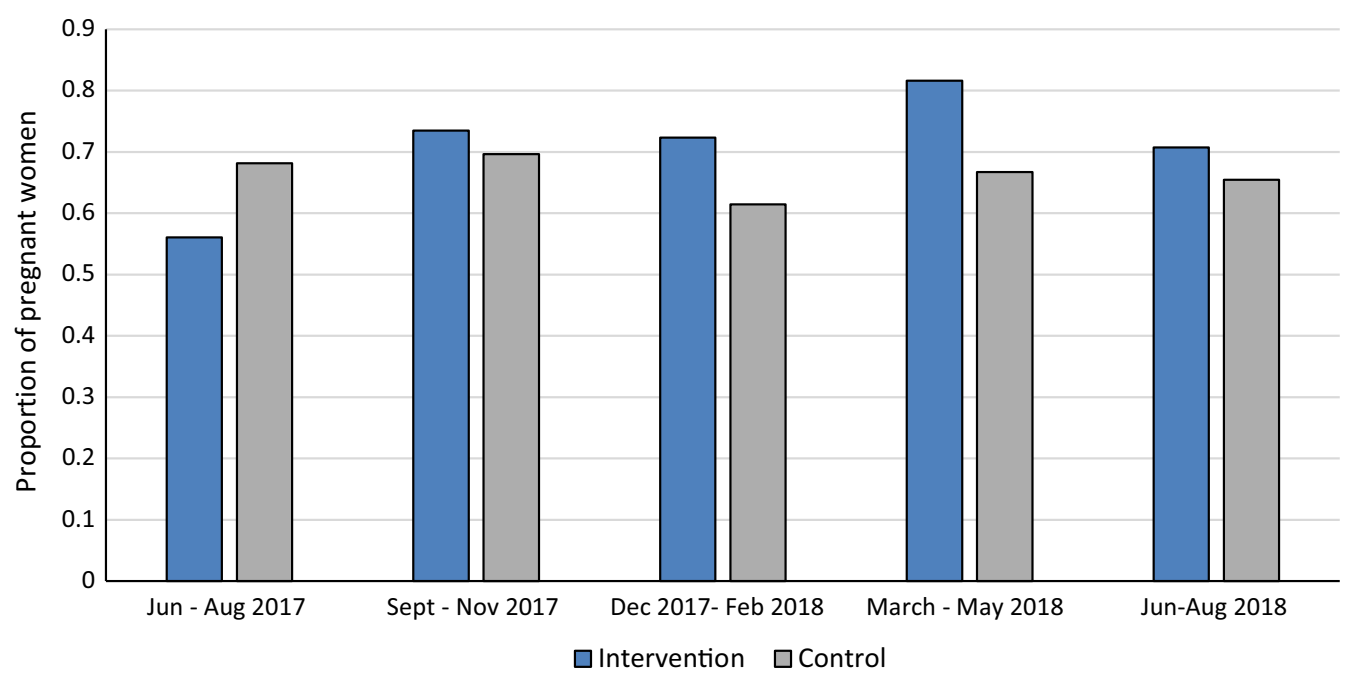

Fig. 3 Proportion of women retained in ANC4 (ANC4/ANC1) by study arm and quarter

\section{Discussion}

Despite a WHO recommendation for all pregnant women to receive at least three doses of IPTp, coverage in most of sub-Saharan Africa remains below international targets [6]. This study demonstrates that CHWs can effectively deliver IPTp, without adversely affecting ANC attendance. Further, not only did community delivery of IPTp not lead to a reduction in ANC attendance, but retention in ANC improved over the course of the study, with 12.6 percentage point increase in ANC4 in the intervention arm compared to the control arm $(95 \%$ CI - 7.0, 32.2). More than half of the IPTp doses were delivered by the CHWs, highlighting their substantial contribution to the increased coverage. The increase in coverage of ANC4 further highlights the important community education and outreach role of these workers.

Concerns have been raised that providing IPTp in the community could lead to women receiving many more IPTp doses than are currently recommended. By requiring women receive the first dose of IPTp-SP at the HF, this study ensured that women were not started on IPTp until they were deemed eligible, at least 13 weeks. Prior to implementation of the study, a small proportion of women reported that they had received 6 doses of IPTp. At endline, there was a small and insignificant increase in women receiving 6 doses, and only a very few women $(\mathrm{n}=6)$ who reported having received 7 doses, highlighting that IPTp was being correctly given according to the monthly schedule. Further, it was apparent from review of the CHW monthly data that most women had delivered by the time they were due for IPTp5. As the card only had space to record five doses of IPTp, it is possible that the discrepancy in self-reported and documented
IPTp truly reflects that women were receiving six and seven doses-a woman who received her first dose at 13 weeks and then continued to receive doses every 4 weeks thereafter could reach as many as eight doses.

Despite the substantial increases in IPTp coverage, there was not a statistically significant $\mathrm{DiD}$ for any of the measures. This was likely due to insufficient power, as the study was powered on prior estimates of IPTp coverage that were lower than what existed at the time the study began, and assumed a lower ICC than existed. However, the fact that there was an improvement in retention in ANC should alleviate many of the concerns around community delivery of IPTp. While further, larger studies are warranted to confirm that this method of delivery results in significant improvements, this study suggests that there is no disadvantage to community IPTp distribution, with no adverse events reported as a result of SP administration by CHWs.

The importance of early attendance at ANC cannot be overstated. Late attendance at ANC has been associated with an increased risk of maternal and fetal complications, including low birth weight, premature delivery, and stillbirth $[17,18]$. Early initiation of ANC is crucial to ensure pregnant women receive health education and preventive services, such as iron supplementation, tetanus injection, and deworming, as well as early implementation of IPTp, which has been shown to have a greater impact than later administration [19-22]. Engaging CHWs in identifying pregnant women in the community and encouraging them to attend ANC early has the potential to improve coverage of early ANC. At endline, women in the intervention arm started ANC significantly earlier than women 
Table 2 Sociodemographic characteristics of baseline and endline survey respondents

\begin{tabular}{|c|c|c|c|c|}
\hline \multirow[t]{3}{*}{ Variable } & \multicolumn{2}{|c|}{ Baseline survey } & \multicolumn{2}{|c|}{ Endline survey } \\
\hline & Intervention & Control & Intervention & Control \\
\hline & $N=188$ & $N=186$ & $N=180$ & $N=180$ \\
\hline \multicolumn{5}{|l|}{ District } \\
\hline Batie, n (\%) & $60(32.3)$ & $60(31.91)$ & $60(33.3)$ & $60(33.3)$ \\
\hline Ouargaye, n (\%) & $64(34.4)$ & $69(36.7)$ & $60(33.3)$ & $60(33.3)$ \\
\hline Po, n (\%) & $62(33.3)$ & $59(31.4)$ & $60(33.3)$ & $60(33.3)$ \\
\hline \multicolumn{5}{|l|}{ Age, in years } \\
\hline Median (Range) & $26(18,44)$ & $27(18,47)$ & $27(18,45)$ & $28(18,48)$ \\
\hline$<20, \mathrm{n}(\%)$ & $26(14.0)$ & $28(15.0)$ & $25(13.9)$ & $21(11.7)$ \\
\hline $20-24, \mathrm{n}(\%)$ & $47(25.3)$ & $51(27.1)$ & $50(27.8)$ & $45(25.0)$ \\
\hline $25-29, \mathrm{n}(\%)$ & $44(23.7)$ & $42(22.3)$ & $33(18.3)$ & $35(19.4)$ \\
\hline $30-34$ & $39(21.0)$ & $34(18.1)$ & $41(22.8)$ & $35(19.4)$ \\
\hline$\geq 35, \mathrm{n}(\%)$ & $30(16.1)$ & $33(17.6)$ & $31(17.2)$ & $44(24.4)$ \\
\hline \multicolumn{5}{|l|}{ Education } \\
\hline None/only religious school, n (\%) & $130(69.1)$ & $109(58.6)$ & $102(56.7)$ & $126(70.0)$ \\
\hline Primary/literate, n (\%) & $43(22.9)$ & $51(27.4)$ & $64(35.6)$ & $33(18.3)$ \\
\hline Secondary or higher, n (\%) & $23(12.2)$ & $28(15.1)$ & $14(7.8)$ & $21(11.7)$ \\
\hline \multicolumn{5}{|l|}{ Gravidity } \\
\hline $1, \mathrm{n}(\%)$ & $42(22.8)$ & $53(28.3)$ & $41(22.8)$ & $39(21.7)$ \\
\hline $2, \mathrm{n}(\%)$ & $24(13.0)$ & $33(17.6)$ & $37(20.6)$ & $28(15.6)$ \\
\hline $3, \mathrm{n}(\%)$ & $34(18.5)$ & $26(13.9)$ & $25(13.9)$ & $28(15.6)$ \\
\hline $4, \mathrm{n}(\%)$ & $42(22.8)$ & $25(13.4)$ & $20(11.1)$ & $22(12.2)$ \\
\hline $5+, n(\%)$ & $42(22.8)$ & $50(26.7)$ & $57(31.7)$ & $63(35.0)$ \\
\hline Married, n (\%) & $180(96.8)$ & $179(95.2)$ & $175(97.2)$ & $169(93.9)$ \\
\hline \multicolumn{5}{|l|}{ Religion } \\
\hline Muslim, n (\%) & $77(41.4)$ & $73(38.8)$ & $73(40.6)$ & $62(34.4)$ \\
\hline n (\%) & $39(21.0)$ & $24(12.8)$ & $38(21.1)$ & $24(13.3)$ \\
\hline Protestant, n (\%) & $15(8.1)$ & $36(19.2)$ & 34 (18.9) & $42(23.3)$ \\
\hline Traditional/animist, n (\%) & $45(24.2)$ & $51(27.1)$ & $28(15.6)$ & $48(26.7)$ \\
\hline None, n (\%) & $10(5.4)$ & $4(2.1)$ & $7(3.9)$ & $4(2.2)$ \\
\hline Work outside the home, n (\%) & $85(45.7)$ & $87(46.3)$ & $88(48.9)$ & $86(47.8)$ \\
\hline
\end{tabular}

in the control arm, suggesting an impact of the intervention. While a large proportion of women spoke to a CHW about their pregnancy, there is still room for improvement, as even in the intervention area, $24 \%$ of women reportedly spoke with neither; engaging these women could have substantially boosted coverage.

Our results suggest that women recognize very early in pregnancy that they are pregnant, and that they disclose their pregnancies most often to their husbands or other family members. This suggests that messaging around the importance of attending ANC early needs to target not only the pregnant woman, but also their husbands and families, to encourage them to attend early, as has been suggested previously [23-25]. In Ethiopia, male involvement in ANC was positively associated with both ANC attendance in first trimester and delivery in a HF [24].

c-IPTp may be a promising new channel for scaling up and closing the remaining coverage gap of IPTp, particularly in remote rural areas and in high malaria burden countries. WHO recognizes that while some countries have experienced a reduced burden of malaria, progress in high-burden countries such as Burkina Faso has stalled in recent years. In 2016, there were an estimated 216 million cases of malaria globally, marking a return to 2012 case levels, with an increase to 219 million cases in 2017 [6, 26]. WHO points to persistent coverage gaps of proven prevention and control interventions in high burden countries, due in part to weak health systems. According to WHO's High-Burden to High Impact 
Table 3 ANC and IPTp coverage at baseline and endline as documented on the ANC card, by study arm

\begin{tabular}{|c|c|c|c|c|c|c|}
\hline & \multicolumn{2}{|l|}{ Baseline } & \multicolumn{2}{|l|}{ Endline } & \multirow[t]{3}{*}{ Difference in differences ${ }^{a}$} & \multirow[t]{3}{*}{$p$-value for DiD } \\
\hline & Control & Intervention & Control & Intervention & & \\
\hline & $N=188$ & $N=186$ & $N=180$ & $N=180$ & & \\
\hline $\begin{array}{l}\text { Number of IPTp doses (mean, } \\
95 \% \mathrm{Cl} \text { ) }\end{array}$ & $2.3(1.9,2.8)$ & $2.3(1.8,2.9)$ & $2.1(1.53,2.9)$ & $2.9(2.3,3.8)$ & $0.9(-0.3,2.1)$ & 0.15 \\
\hline IPTp1+ (\%, 95\% Cl) & $86.2(73.5,93.4)$ & $80.7(65.4,90.2)$ & $75.6(54.6,88.8)$ & $86.1(74.9,92.8)$ & $16.1 \%$-points $(-8.5,40.7)$ & 0.20 \\
\hline IPTp2+ (\%, 95\% CI) & $73.4(55.2,86.1)$ & $69.9(57.1,80.2)$ & $64.4(41.3,82.3)$ & $72.2(50.3,87.0)$ & $11.3 \%$-points $(-27.6,50.1)$ & 0.57 \\
\hline IPTp3+ (\%, 95\% CI) & $54.3(39.3,68.5)$ & $50.5(35.0,66.0)$ & $47.2(28.6,66.7)$ & $61.1(40.6,78.4)$ & $17.6 \%$-points $(-16.3,51.5)$ & 0.31 \\
\hline IPTp4+ (\%, 95\% CI) & $16.0(8.4,28.3)$ & $21.5(8.3,45.3)$ & $21.1(12,34.5)$ & $46.7(29.6,64.6)$ & $20.0 \%$-points $(-7.2,47.3)$ & 0.15 \\
\hline IPTp5+ (\%, 95\% CI) & $2.7(1.0,6.8)$ & $4.3(1.1,15.4)$ & $2.2(0.6,8.1)$ & $27.8(17.3,41.4)$ & $23.9 \%$-points $(9.4,38.4)$ & 0.001 \\
\hline $\begin{array}{l}\text { Number of ANC visits (mean, } \\
95 \% \mathrm{Cl} \text { ) }\end{array}$ & $3.2(2.9,3.6)$ & $3.23(2.9,3.6)$ & $3.4(3.0,3.7)$ & $3.6(3.6,3.7)$ & $0.2(-0.3,0.8)$ & 0.43 \\
\hline ANC1+ $(\%, 95 \%$ Cl) & $89.4(80.4,94.5)$ & $90.3(76.8,96.4)$ & $94.4(84.7,98.1)$ & $97.8(94.6,99.1)$ & $2.4 \%$-points $(-10.7,15.5)$ & 0.72 \\
\hline ANC4+ (\%, 95\% Cl) & $62.2(49.4,73.5)$ & $61.8(50.5,72.0)$ & $65.0(48.5,78.6)$ & $77.2(73.1,80.9)$ & $12.6 \%$-points $(-7.0,3.22)$ & 0.21 \\
\hline
\end{tabular}

IPTp intermittent preventive treatment in pregnancy, ANC antenatal care visits

a Confidence interval for DiD were estimated with identity link and either binomial or poisson distribution, as appropriate

Table 4 ANC and IPTp coverage at baseline and endline, by study arm, adjusted for gravidity and maternal age

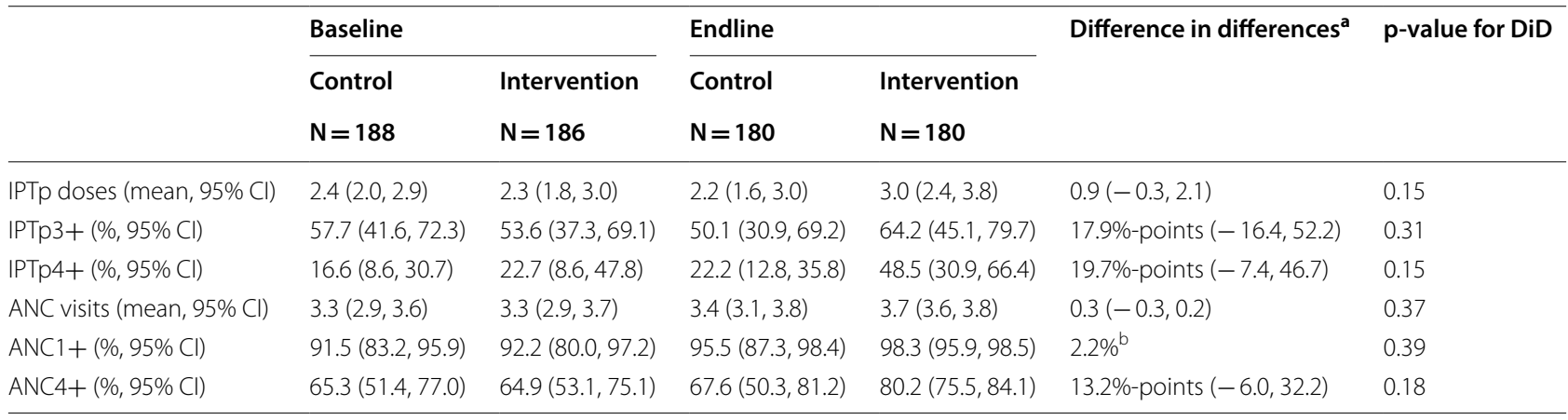

IPTp Intermittent preventive treatment in pregnancy; ANC antenatal care visits

a Results calculated using a logistic regression model with an interaction term for survey and arm, adjusted for gravidity and maternal age ( $<20$ vs $\geq 20$ years); Confidence interval for DiD were estimated with identity link and either binomial or poisson distribution, as appropriate

b The identity link model for ANC1+ did not converge, thus is was not possible to calculate a $95 \% \mathrm{CI}$

Initiative: national data from many high-burden countries show coverage gaps for the core malaria interventions (i.e. vector control, case management, and IPTp) [27]. In 2016, approximately $80 \%$ of eligible pregnant women across sub-Saharan Africa did not receive the recommended three or more doses of IPTp [27]. Implementation of c-IPTp could reduce the number of pregnant women in sub-Saharan Africa who are not offered the third dose of IPTp during their pregnancy.

While c-IPTp is not the only solution to improving IPTp uptake, it has several advantages, including shifting the task of providing IPTp from already overburdened ANC facility staff to trained CHWs, allowing ANC nurses to spend more time with their clients providing a positive pregnancy experience-a key recommendation of the 2016 WHO guideline on ANC, providing "pregnant women with respectful, individualized, person-centred care at every contact" [28]. While task-shifting components of ANC health promotion to a broad range of cadres, the WHO recommendation for delivery of IPTp falls short of recommending task-shifting to lay health workers, including only "auxiliary nurses, nurses, midwives and doctors." This study's findings suggest that WHO's recommendations on task shifting of IPTp could be expanded to include lay health workers; offering an alternative approach that increases patient access to services and enhances efficiency of the ANC services package. Ample data exist from malaria diagnosis and treatment studies highlighting CHW feasibility and capabilities in effectively providing malaria treatment, accurately 
Table 5 Effects of gravidity and maternal age on uptake of IPTp and ANC

\begin{tabular}{llll}
\hline & OR & Confidence limits & p-value \\
\hline IPTp doses & & & \\
$\quad$ Gravidity, Primi vs multi & 0.84 & $(0.76,0.94)$ & 0.002 \\
$\quad$ Age $<20$ & 1.02 & $(0.86,1.21)$ & 0.78 \\
IPTp3 & & & \\
Gravidity, Primi vs multi & 0.62 & $(0.39,0.99)$ & 0.05 \\
Age $<20$ & 0.93 & $(0.49,1.77)$ & 0.83 \\
IPTp4 & & & \\
Gravidity, Primi vs multi & 0.71 & $(0.43,1.17)$ & 0.18 \\
Age $<20$ & 1.02 & $(0.65,1.59)$ & 0.94 \\
ANC visits & & & \\
Gravidity, Primi vs multi & 0.98 & $(0.9,1.06)$ & 0.64 \\
Age $<20$ & 0.92 & $(0.87,0.99)$ & 0.02 \\
ANC1 & & & 0.37 \\
Gravidity, Primi vs multi & 0.73 & $(0.36,1.46)$ & 0.03 \\
Age $<20$ & 0.58 & $(0.36,0.94)$ & \\
ANC4 & & & 0.75 \\
Gravidity, Primi vs multi & 0.91 & $(0.51,1.61)$ & 0.02 \\
Age $<20$ & 0.61 & $(0.4,0.93)$ & \\
\hline
\end{tabular}

diagnosing fever following RDT results, and providing seasonal malaria chemoprophylaxis to children under 5 years of age [29-31]. Several studies, including this one, demonstrated that $\mathrm{CHWs}$ can contribute to improved ANC attendance and ANC attendance is not negatively affected by community delivery of IPTp, with no reported increases in adverse events $[7,8,11]$.

\section{Conclusions}

To achieve the ambitious targets for malaria eradication by 2050 [32], rapid accelerated coverage of proven interventions is needed. Every available tool in our toolbox must be used, including engaging communities in this effort and allowing CHWs to deliver malaria prevention and control interventions. After decades of promoting IPTp, coverage of this intervention remains among the lowest, with an estimated $22 \%$ of pregnant women receiving the recommended three doses [6]. Implementation of community-IPTp provided by trained CHWs could improve coverage and access for millions of pregnant women across sub-Saharan Africa. With an estimated 215,000 LBW deliveries annually as well as up to $20 \%$ of stillbirths in sub-Saharan Africa which could be prevented by complete scale-up of IPTp, the time to act is now $[33,34]$.

\section{Abbreviations}

ANC: Antenatal care; CHW: Community health worker; Cl: Confidence interval; c-IPTp: Community level distribution of intermittent preventive treatment of pregnant women; DiD: Difference in differences; HF: Health facility; IPTp: Intermittent preventive treatment of pregnant women; LLIN: Long-lasting insecticide-treated nets; MIP: Malaria in pregnancy; $\mathrm{MOH}$ : Ministry of Health; SP: Sulfadoxine pyrimethamine; WHO: World Health Organization.

\section{Acknowledgements}

The authors would like to thank all the community and facility health workers who participated in this study. We would like to thank Edward Bunker for his assistance overseeing data collection and Dr. Gayane Yenokyan for her assistance with data analyses.

\section{Disclaimer}

The findings and conclusions in this paper are those of the authors and do not necessarily represent the views of the U.S. Centers for Disease Control and Prevention or the US Agency for International Development.

\section{Authors' contributions}

JRG, WRB, SJY, and DB conceived of the study with input from JT, OB, MT, and YS. DB and WRB wrote the protocol with input from JRG. WRB, JT, OB, MD, DB, $Y S$, and WRB conducted the training and oversaw the data collection. JRG, DKS, and WRB analysed data, with input from JW. JRG drafted the manuscript. $D B, J W, K V, S J Y$, and WRB provided critical intellectual feedback and assisted in revising the manuscript. All authors read and approved the final manuscript.

\section{Funding}

This study is made possible by the generous support of the American people through the United States Agency for International Development (USAID) under the terms of the Cooperative Agreement AID-OAA-A-14-00028. The contents are the responsibility of the authors and do not necessarily reflect the views of USAID or the United States Government.

\section{Availability of data and materials}

Data has been made available on the USAID Development Data Library (DDL; https://data.usaid.gov/) as restricted access pending approval from Burkina Faso government. Data can also be requested from Jhpiego by writing to opendatahelp@jhpiego.org under a data use agreement.

\section{Ethics approval and consent to participate}

This study was approved by the Johns Hopkins Bloomberg School of Public Health and Burkina Faso IRBs. CDC staff participated under a non-engaged determination from the CDC Human Subjects Research Protection Office. Women provided verbal consent to receive care from a CHW; written informed consent was obtained from all participants in the cross-sectional survey prior to participation.

\section{Consent for publication}

Not applicable.

\section{Competing interests}

The authors declare that they have no competing interests.

\section{Author details}

${ }^{1}$ Malaria Branch, Division of Parasitic Diseases and Malaria, Center for Global Health, US Centers for Disease Control and Prevention, 1600 Clifton Road, Mailstop H24-3, Atlanta, GA 30329, USA. ${ }^{2}$ The Johns Hopkins Bloomberg School of Public Health, Johns Hopkins University, Baltimore, MD, USA. ${ }^{3}$ Jhpiego, Ouagadougou, Burkina Faso. ${ }^{4}$ Jhpiego, Baltimore, MD, USA. ${ }^{5}$ US President's Malaria Initiative, US Agency for International Development, Washington, D.C., USA. ${ }^{6}$ National Malaria Control Programme, Ministry of Health, Ouagadougou, Burkina Faso.

Received: 2 April 2020 Accepted: 29 July 2020

Published online: 05 August 2020

References

1. Desai M, ter Kuile FO, Nosten F, McGready R, Asamoa K, Brabin B. Epidemiology and burden of malaria in pregnancy. Lancet Infect Dis. 2007;7:93-104. 
2. WHO. Updated $\mathrm{WHO}$ policy recommendation: intermittent preventive treatment of malaria in pregnancy using sulfadoxine-pyrimethamine (IPTp-SP). Geneva: World Health Organization; 2012. http://www.who. int/entity/malaria/publications/atoz/who_iptp_sp_policy_recommenda tion/en/index.html.

3. WHO. Malaria in pregnant women. Geneva: World Health Organization. https://www.who.int/malaria/areas/high_risk_groups/pregnancy/en/.

4. World Health Organization. World malaria report 2011. Geneva: World Health Organization; 2011

5. WHO. Policy brief for the implementation of intermittent preventive treatment of malaria in pregnancy using sulfadoxine-pyrimethamine (IPTp-SP). Geneva: World Health Organization; 2013. https://www.who. int/malaria/publications/atoz/policy_brief_iptp_sp_policy_recommenda tion/en/.

6. WHO. World malaria report 2018. Geneva: World Health Organization; 2018

7. Okeibunor JC, Orji BC, Brieger W, Ishola G, Otolorin E, Rawlins B. Preventing malaria in pregnancy through community-directed interventions: evidence from Akwa Ibom State, Nigeria. Malar J. 2011;10:227.

8. Ndyomugyenyi R, Clarke SE, Hutchison CL, Hansen KS, Magnussen P. Efficacy of malaria prevention during pregnancy in an area of low and unstable transmission: an individually-randomised placebo-controlled trial using intermittent preventive treatment and insecticide-treated nets in the Kabale Highlands, southwestern Uganda. Trans R Soc Trop Med Hyg. 2011;105:607-16

9. Mbonye AK, Magnussen P, Bygbjerg IB. Intermittent preventive treatment of malaria in pregnancy: the effect of new delivery approaches on access and compliance rates in Uganda. Trop Med Int Health. 2007;12:519-31.

10. Msyamboza KP, Savage EJ, Kazembe PN, Gies S, Kalanda G, D'Alessandro $U$, et al. Community-based distribution of sulfadoxine-pyrimethamine for intermittent preventive treatment of malaria during pregnancy improved coverage but reduced antenatal attendance in southern Malawi. Trop Med Int Health. 2009:14:183-9.

11. Gies S, Coulibaly SO, Ky C, Ouattara FT, Brabin BJ, D'Alessandro U. Community-based promotional campaign to improve uptake of intermittent preventive antimalarial treatment in pregnancy in Burkina Faso. Am J Trop Med Hyg. 2009;80:460-9

12. Institut National de la Statistique et de la Démographie (INSD), Programme d'Appui au Développement Sanitaire (PADS), Programme National de Lutte contre le Paludisme (PNLP), et ICF: Enquête sur les indicateurs du paludisme au Burkina Faso, 2017-2018. Rockville: INSD, PADS, PNLP et ICF; 2018

13. Institut National de la Statistique et de la Démographie (INSD) et ICF International: Enquête Démographique et de Santé et à Indicateurs Multiples du Burkina Faso 2010. Calverton: INSD and ICF International; 2012.

14. WHO. High burden to high impact: a targeted malaria response. WHO/ CDS/GMP/2018.25. Rev.1. Geneva: World Health Organization; 2019. https ://apps.who.int/iris/bitstream/handle/10665/275868/WHO-CDS-GMP2018.25-eng.pdf?ua=1. Accessed 19 Oct 2019.

15. Ministère de la Santé Burkina Faso. Directives nationales pour la prise en charge du paludisme dans les formations sanitaires du Burkina Faso. Version révisée mars 2014.

16. WHO. Training for mid-level managers (MLM) - WHO/IVB/08.07 Module 7: The EPI coverage survey. Geneva: World Health Organization; 2008.

17. Beauclair R, Petro G, Myer $L$. The association between timing of initiation of antenatal care and stillbirths: a retrospective cohort study of pregnant women in Cape Town. South Africa. BMC Pregnancy Childbirth. 2014:14:204.

18. Heaman MI, Newburn-Cook CV, Green CG, Elliott L, Helewa ME. Inadequate prenatal care and its association with adverse pregnancy outcomes: a comparison of indices. BMC Pregnancy Childbirth. 2008;8:15.
19. Huynh B, Fievet N, Briand V, Borgella S, Massougbodji A, Deloron P, et al. Consequences of gestational malaria on birth weight: finding the best timeframe for intermittent preventive treatment administration. PLoS ONE. 2012;7:e35342.

20. Huynh B, Fievet N, Gbaguidi G, Dechavanne S, Borgella S, Guezo-Mevo $B$, et al. Influence of the timing of malaria infection during pregnancy on birth weight and on maternal anemia in Benin. Am J Trop Med Hyg. 2011;85:214-20.

21. Valea I, Tinto H, Drabo MK, Huybregts L, Sorgho H, Ouedraogo JB, et al. An analysis of timing and frequency of malaria infection during pregnancy in relation to the risk of low birth weight, anaemia and perinatal mortality in Burkina Faso. Malar J. 2012;11:71.

22. Paudel YR, Jha T, Mehata S. Timing of First Antenatal Care (ANC) and Inequalities in Early Initiation of ANC in Nepal. 2017;5.

23. Simkhada B, Porter MA, van Teijlingen ER. The role of mothers-in-law in antenatal care decision-making in Nepal: a qualitative study. BMC Pregnancy Childbirth. 2010;10:34.

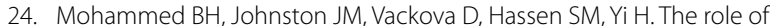
male partner in utilization of maternal health care services in Ethiopia: a community-based couple study. BMC Pregnancy Childbirth. 2019;19:28.

25. Hill J, Kayentao K, Achieng F, Diarra S, Dellicour S, Diawara SI, et al. Access and use of interventions to prevent and treat malaria among pregnant women in Kenya and Mali: a qualitative study. PLOS ONE. 2015;10:e0119848.

26. WHO. World malaria report 2017. Geneva: World Health Organization; 2017.

27. WHO. $10+1$ Approach-an intensified effort to reduce malaria cases and deaths: Getting back on track to achieve the morbidity and mortality reduction targets of the Global Technical Strategy for Malaria 2016-2030; Background document for Session 5 of the Malaria Policy Advisory Committee Meeting; 17-19 October 2018, Geneva, Switzerland. https://www. who.int/malaria/mpac/mpac-october2018-session5-10plus1-approach. pdf. Accessed 19 Oct 2019.

28. WHO. WHO recommendations on antenatal care for a positive pregnancy experience. Geneva: World Health Organization; 2016.

29. Singlovic J, Ajayi IO, Nsungwa-Sabiiti J, Siribié M, Sanou AK, Jegede AS, et al. Compliance with malaria rapid diagnostic testing by community health workers in 3 malaria-endemic countries of sub-Saharan Africa: an observational study. Clin Infect Dis. 2016;63:S276-82.

30. Boyce MR, Menya D, Turner EL, Laktabai J, Prudhomme-O'Meara W. Evaluation of malaria rapid diagnostic test (RDT) use by community health workers: a longitudinal study in western Kenya. Malar J. 2018;17:206.

31. Smith Paintain L, Willey B, Kedenge S, Sharkey A, Kim J, Buj V, et al. Community health workers and stand-alone or integrated case management of malaria: a systematic literature review. Am J Trop Med Hyg. 2014;91:461-70.

32. Feachem RGA, Chen I, Akbari O, Bertozzi-Villa A, Bhatt S, Binka F, et al. Malaria eradication within a generation: ambitious, achievable, and necessary. Lancet. 2019;394:1056-112.

33. Lawn JE, Blencowe H, Waiswa P, Amouzou A, Mathers C, Hogan D, et al. Stillbirths: rates, risk factors, and acceleration towards 2030. Lancet. 2016;387:587-603.

34. Walker PGT, ter Kuile FO, Garske T, Menendez C, Ghani AC. Estimated risk of placental infection and low birthweight attributable to Plasmodium falciparum malaria in Africa in 2010: a modelling study. Lancet Glob Health. 2014:2:e460-7.

\section{Publisher's Note}

Springer Nature remains neutral with regard to jurisdictional claims in published maps and institutional affiliations. 